\title{
Cohomological Intersection Numbers for the Generalized Airy Functions at Veronese Points
}

\author{
By \\ Irina Basalaeva, Hironobu Kimura* and Takashi NakazukA \\ (Kumamoto University, Japan)
}

\begin{abstract}
We establish the exterior power structure for the polynomial twisted de Rham cohomology group associated with the generalized Airy functions at a point of extended Veronese variety. Using this structure, we obtain a natural basis of the twisted de Rham cohomology group from that of one dimensional case, which is considered as an analogue of the flat basis of the Jacobi ring of $A$-type simple singularity. We compute explicitly the cohomological intersection numbers for these basis and extend the result of Iwasaki and Matsumoto.

Keywords and Phrases. Generalized Airy function, Intersection number, Flat basis, Generalized Veronese variety, Twisted de Rham cohomology.

2000 Mathematics Subject Classification Numbers. 33C70.
\end{abstract}

\section{Introduction}

For the Gamma and Euler's Beta functions, the following formulas are well known.

$$
\begin{gathered}
\Gamma(a) \Gamma(1-a)=\frac{\pi}{\sin \pi a} \\
\left(\frac{1}{a}+\frac{1}{b}\right) B(a, b) B(1-a, 1-b)=-2 \pi i \frac{e^{2 \pi i(a+b)}-1}{\left(e^{2 \pi i(a)}-1\right)\left(e^{2 \pi i(b)}-1\right)} .
\end{gathered}
$$

Similar formulas are known for the Gauss hyperegeometric function or its confluent family: Kummer's confluent hypergeometric function, Bessel function, Hermite-Weber function and Airy function [19]. These formulas contain terms of product of the special functions with different parameters, like as $B(a, b) B(1-a, 1-b)$ for the Beta function, and therefore they are called the quadratic relations.

In this paper, aiming at establishing a quadratic relation for the generalized Airy function, we compute the cohomological intersection numbers for this function.

\footnotetext{
* Partly supported by Grant-in Aid for Scientific Research (B-11440058), the Ministry of Education, Science and Culture, Japan.
} 
We shall explain what the generalized Airy function is and how the computation of the cohomological intersection numbers may relate to the quadratic relations for the known cases.

The generalized Airy function, introduced by Gelfand et al. [6], is defined by the integral of the form

$$
\int_{\Delta(z)} e^{f(x, z, \alpha)} d x_{1} \wedge \cdots \wedge d x_{n}
$$

where $f$ is the polynomial of $x$ given by (2.4) depending on matrices $z$ in the set

$$
\begin{aligned}
Z_{n+1}=\left\{\left(z_{0}, \ldots, z_{N+1}\right) \in M(n+1, N+2, \boldsymbol{C}) \mid\right. \\
\\
\left.\operatorname{det}\left(z_{0}, \ldots, z_{n}\right) \neq 0, z_{0}={ }^{t}(1,0, \ldots, 0)\right\},
\end{aligned}
$$

and $\Delta(z)$ is, roughly speaking, a locally finite $n$-cycle on which $\Re(f)$ tends to $-\infty$ faster than $-|x|^{q}$ for some positive number $q$ as $|x| \rightarrow \infty$. See Section 2 about the details of the definition of the geralized Airy function and see also [15], [22], [23] for the cycles. In this context, the classical Airy function

$$
\operatorname{Ai}(\zeta)=\int_{\Delta} e^{x \zeta-x^{3} / 3} d x
$$

corresponds to the case $(n, N)=(1,2)$ with

$$
z=\left(\begin{array}{llll}
1 & 0 & 0 & 0 \\
0 & 1 & 0 & \zeta
\end{array}\right) .
$$

The generalized Airy function and the classical special functions mentioned above are understood in a unified way in the framework of the general hypergeometric functions (GHF, for short) ([16]). For a partition $\lambda$ of $N+2$, we have the maximal abelian subgroup $H_{\lambda}$ of $\operatorname{GL}(N+2, C)$ which is the centralizer of a regular element of Jordan normal form indexed by a partition $\lambda$ of $N+2$. The GHF of type $\lambda$ is a function on a Zariski open subset of $M(n+1, N+2 ; \boldsymbol{C})$ defined as a Radon transform by $n$-dimensional integral of a character of the universal covering group of $H_{\lambda}$. The Beta function and the Gamma function correspond to the case $(n, N)=(1,1)$ with the partitions $(1,1,1)$ and $(2,1)$ of 3 , respectively. And the Gauss hypergeometric function, Kummer's confluent hypergeometric function, Bessel function, Hermite-Weber function and Airy function correspond to the case $(n, N)=(1,2)$ with the partitions $(1,1,1,1),(2,1,1),(2,2),(3,1)$ and (4) respectively. For the case $(n, N)$ general, the partitions $(1, \ldots, 1)$ and $(N+2)$ correspond to Aomoto-Gelfand hypergeometric function and the generalized Airy function, respectively $([1,5])$.

The quadratic relations for the GHF's are studied for the case of $n=1$ ([19]) and for Aomoto-Gelfand hypergeometric function ([21]). One of the method of obtaining the quadratic relations for these cases is to use the in- 
tersection theory for the twisted de Rham cohomology and of twisted homology. We can define the twisted de Rham cohomology group $H_{+}^{n}$ for the GHF and its dual cohomology group $H_{-}^{n}$, and we can also define the twisted homology group $H_{n}^{+}$and its dual group $H_{n}^{-}$. If one take base $\left\{\varphi_{i}^{+}\right\}$and $\left\{\varphi_{i}^{-}\right\}$ for the cohomology groups $H_{+}^{n}$ and $H_{-}^{n}$, respectively, and if one take base $\left\{\gamma_{i}^{+}\right\}$ and $\left\{\gamma_{i}^{-}\right\}$for the homology groups $H_{n}^{+}$and $H_{n}^{-}$, respectively, we can define the hypergeometric period matrices

$$
M_{+}=\left(\int_{\gamma_{j}^{+}} U \varphi_{i}^{+}\right)_{i, j}, \quad M_{-}=\left(\int_{\gamma_{j}^{-}} U^{-1} \varphi_{i}^{-}\right)_{i, j}
$$

and the cohomological and homological intersection matrices

$$
I_{h}=\left(\left\langle\gamma_{i}^{+}, \gamma_{j}^{-}\right\rangle\right), \quad I_{c}=\left(\left\langle\varphi_{i}^{+}, \varphi_{j}^{-}\right\rangle\right) .
$$

Here, $U$ is the function which appears as the integrand of the integral representation of the GHF. For example in the Gauss' case, $U=$ $u^{a-1}(1-u)^{c-a-1}(1-x u)^{-b}$. For these matrices we have the relation

$$
{ }^{t} M_{-}\left(I_{h}\right)^{-1} M_{+}=(2 \pi)^{n} \sqrt{-1} t I_{c},
$$

which yields the quadratic relations.

So if one try to establish the quadratic relations for the GHF's along this line of thought, it is important to give the explicit form of the intersection matrices $I_{h}$ and $I_{c}$ for appropriately chosen bases of cohomology and homology groups. This is just what we shall carry out in this paper for the cohomology group of the generalized Airy function.

We explain the outline of this paper.

In Section 2, we review the definition of the generalized Airy function. For any fixed $z \in Z_{n+1}$ and the polynomial $f$ given by (2.4), the twisted de Rham complex $\left(\Omega^{\bullet}(X), d_{f}\right)$ is defined as a pair of the set $\Omega^{\bullet}(X)$ of polynomial differential forms on $X=C^{n}$ and the twisted exterior differentiation

$$
d_{f} \eta=\left(e^{-f} \cdot d \cdot e^{f}\right) \eta=d \eta+d f \wedge \eta, \quad \eta \in \Omega^{\bullet}(X) .
$$

The twisted cohomology group $H^{n}\left(\Omega^{\bullet}(X), d_{f}\right)$ is the cohomology group of the complex $\left(\Omega^{\bullet}(X), d_{f}\right)$. Similarly we define the cohomology group $H^{n}\left(\Omega^{\bullet}(X)\right.$, $\left.d_{-f}\right)$. It is known that $\operatorname{dim}_{C} H^{n}\left(\Omega^{\bullet}(X), d_{f}\right)=\operatorname{dim}_{C} H^{n}\left(\Omega^{\bullet}(X), d_{-f}\right)=\left(\begin{array}{c}N \\ n\end{array}\right)$ for any $z \in Z_{n+1}$ and base for these groups can be explicitly given (Proposition 3.2). In Section 4, the exterior power structure for the cohomology groups $H^{n}\left(\Omega^{\bullet}(X), d_{ \pm f}\right)$ is studied when $z$ is a point of a subvariety of $Z_{n+1}$ of dimension $2(N+1)$ called the generalized Veronese variety. In Section 5, we review the result due to Iwasaki ([9]) on the duality between the polynomial twisted cohomologies:

$$
H^{n}\left(\Omega^{\bullet}(X), d_{f}\right) \times H^{n}\left(\Omega^{\bullet}(X), d_{-f}\right) \rightarrow C,
$$


see Theorem 5.1. This duality gives a complex number $\left\langle\phi^{+}, \phi^{-}\right\rangle$for $\phi^{+} \in$ $H^{n}\left(\Omega^{\bullet}(X), d_{f}\right)$ and $\phi^{-} \in H^{n}\left(\Omega^{\bullet}(X), d_{-f}\right)$, which is called the cohomological intersection number of $\phi^{+}$and $\phi^{-}$. Then Iwasaki and Matsumoto ([11]) computed the matrix of cohomological intersection numbers for the case $z=$ $\left(I_{n+1}, 0\right) \in Z_{n+1}$. They used a basis of the cohomology group $H^{n}\left(\Omega^{\bullet}(X), d_{f}\right)$ of the form $s_{\mu}(x) d x_{1} \wedge \cdots \wedge d x_{n}$, where $s_{\mu}(x)$ is the polynomial of $x$ such that $s_{\mu}(e(y))$, the function obtained by substituting the elementary symmetric polynomials $e(y)=\left(e_{1}(y), \ldots, e_{n}(y)\right)$ of $y=\left(y_{1}, \ldots, y_{n}\right)$ to $s_{\mu}(x)$, is the Schur function corresponding to the Young diagram $\mu$ with the length $\ell(\mu) \leq n$ and the parts $\mu_{i} \leq N-n$, see Proposition 3.2. We extend their result to the case where $z$ belongs to the generalized Veronese variety (Theorem 5.2). For this extension, we make use of another basis $\left\{\phi_{\mu}\right\}$ of cohomology group $H^{n}\left(\Omega^{\bullet}(X), d_{f}\right)$ given in Proposition 4.10. The forms $\phi_{\mu}$ depend on $z$ and reduce to $s_{\mu}(x) d x_{1} \wedge \cdots \wedge d x_{n}$ when $z=\left(I_{n+1}, 0\right)$. The argument in [11] works also in our case by a slight modification, however we give the proofs for the sake of completeness.

\section{Generalized Airy functions}

In this section, we recall the definition of the generalized Airy function (Airy function, for short). Let $N \geq 1$ be an integer and $H$ the maximal abelian Lie group of the form

$$
H=\left\{\sum_{0 \leq i \leq N+1} h_{i} \Lambda^{i} \mid h_{0} \neq 0, h_{i} \in C\right\} \subset \mathrm{GL}(N+2, C),
$$

where $\Lambda=\left(\delta_{i+1, j}\right)_{0 \leq i, j \leq N+1}$ is the shift matrix. Sometimes it is convenient to write an element $h=\sum_{i} h_{i} \Lambda^{i} \in H$ as $h=\left[h_{0}, h_{1}, \ldots, h_{N+1}\right]$.

The Airy function will be defined as a "Radon transform" of a character of the group $H$. Here a character means a Lie group homomorphism from $H$ to $C^{\times}$. So the characters of $H$ will be described at first. Define the functions $\theta_{i}(h), 0 \leq i \leq N+1$, by the generating function

$$
\log \left(h_{0} I+h_{1} \Lambda+\cdots+h_{N+1} \Lambda^{N+1}\right)=\sum_{i=0}^{N+1} \theta_{i}(h) \Lambda^{i} .
$$

The explicit form of $\theta_{i}$ is obtained as follows. Expand the left hand side of (2.1) as

$$
\begin{aligned}
\log \left(h_{0} I+h_{1} \Lambda+h_{2} \Lambda^{2}+\cdots+h_{N+1} \Lambda^{N+1}\right) \\
=\left(\log h_{0}\right) I+\sum_{m=1}^{\infty} \frac{(-1)^{m+1}}{m}\left(\frac{h_{1}}{h_{0}} \Lambda+\frac{h_{2}}{h_{0}} \Lambda^{2}+\cdots+\frac{h_{N+1}}{h_{0}} \Lambda^{N+1}\right)^{m} .
\end{aligned}
$$


Then $\theta_{0}(h)=\log h_{0}$ and, for $m \geq 1$,

$$
\theta_{m}(h)=\sum_{\substack{k_{1}+2 k_{2}+\cdots+m k_{m}=m \\ k_{1}, \ldots, k_{m} \geq 0}}(-1)^{k_{1}+\cdots+k_{m}-1} \frac{\left(k_{1}+\cdots+k_{m}-1\right) !}{k_{1} ! \ldots k_{n} !}\left(\frac{h_{1}}{h_{0}}\right)^{k_{1}} \ldots\left(\frac{h_{m}}{h_{0}}\right)^{k_{m}} .
$$

We see that the correspondence $H \rightarrow C^{\times} \times C^{N+1}$, defined by

$$
h=\left[h_{0}, h_{1}, \ldots, h_{N+1}\right] \mapsto\left(h_{0}, \theta_{1}(h), \ldots, \theta_{N+1}(h)\right),
$$

gives an isomorphism of the groups ([6]). Hence a character $\chi: H \rightarrow \boldsymbol{C}^{\times}$is given by

$$
\chi(h, \alpha)=h_{0}^{\alpha_{N+1}} \exp \left(\sum_{k=0}^{N} \alpha_{k} \theta_{N-k+1}(h)\right)
$$

for appropriate complex constants $\alpha=\left(\alpha_{0}, \ldots, \alpha_{N+1}\right) \in \boldsymbol{C}^{N+2}$ such that $\alpha_{N+1} \in \boldsymbol{Z}$.

Let $n$ be an integer such that $1 \leq n \leq N$, and let us consider the Radon transform of the character $\chi$ with the condition

$$
\alpha_{N+1}=-n-1 \text {. }
$$

Let $\imath: H \rightarrow C^{\times} \times C^{N+1}$ be the biholomorphic map defined by

and set

$$
\imath\left(\left[h_{0}, h_{1}, \ldots, h_{N+1}\right]\right)=\left(h_{0}, h_{1}, \ldots, h_{N+1}\right)
$$

$$
\begin{aligned}
& Z_{n+1} \\
& =\left\{z=\left(\begin{array}{cccc}
1 & z_{01} & \cdots & z_{0, N+1} \\
0 & z_{11} & \cdots & z_{1, N+1} \\
\vdots & \vdots & & \vdots \\
0 & z_{n 1} & \cdots & z_{n, N+1}
\end{array}\right) \in M(n+1, N+2, C) \mid \operatorname{det}\left(z_{i j}\right)_{0 \leq i, j \leq n} \neq 0\right\} .
\end{aligned}
$$

Let $t=\left(t_{0}, t_{1}, \ldots, t_{n}\right)$ be the homogeneous coordinates of $\boldsymbol{P}^{n}$. For $z=$ $\left(z_{0}, z_{1}, \ldots, z_{N+1}\right) \in Z_{n+1}$, define the vector $t z=\left(t z_{0}, t z_{1}, \ldots, t z_{N+1}\right)$ of $N+2$ linear polynomials in $t$. Then, substitute these polynomials into the character $\chi(\cdot ; \alpha)$ to obtain $\chi\left(l^{-1}(t z) ; \alpha\right)$. By virtue of the assumption $(2.3)$, the $n$-form

$$
\chi\left(l^{-1}(t z) ; \alpha\right) \cdot \tau, \quad \tau=\sum_{i=0}^{n}(-1)^{i} t_{i} d t_{0} \wedge \cdots \wedge d t_{i-1} \wedge d t_{i+1} \wedge \cdots \wedge d t_{n}
$$

is invariant by the homothety $t \mapsto c t\left(c \in C^{\times}\right)$, and hence it is a well defined single valued $n$-form on $\boldsymbol{P}^{n}$ depending holomorphically on $z \in Z_{n+1}$. Notice that the form $\chi\left(l^{-1}(t z) ; \alpha\right) \cdot \tau$ has the singularity along $\left\{t \in \boldsymbol{P}^{n} \mid t_{0}=0\right\}$, since $t z_{0}=t_{0}$. 
Definition 2.1. The generalized Airy function is defined by

$$
F(z, \alpha, \Delta)=\int_{\Delta(z)} \chi\left(l^{-1}(t z) ; \alpha\right) \cdot \tau
$$

where $\Delta(z)$ is some $n$-dimensional homology cycle in $X=\boldsymbol{P}^{n} \backslash\left\{t_{0}=0\right\} \simeq \boldsymbol{C}^{n}$ depending continuously on $z \in Z_{n+1}$. See [15] for the homology group.

Let $x=\left(x_{1}, \ldots, x_{n}\right)$ be the affine coodinates of $X$ defined by $x_{i}=t_{i} / t_{0}$. Then, noticing

$$
\tau=t_{0}^{n+1} d\left(\frac{t_{1}}{t_{0}}\right) \wedge \cdots \wedge d\left(\frac{t_{n}}{t_{0}}\right)
$$

and the assumption (2.3), we see that

$$
\chi\left(l^{-1}(t z) ; \alpha\right) \cdot \tau=\chi\left(l^{-1}(\vec{x} z) ; \alpha\right) d x=e^{f(x, z, \alpha)} d x
$$

with the polynomial

$$
f(x, z, \alpha)=\sum_{k=0}^{N} \alpha_{k} \theta_{N-k+1}\left(l^{-1}(\vec{x} z)\right),
$$

where $\vec{x}=\left(1, x_{1}, \ldots, x_{n}\right)$ and $d x=d x_{1} \wedge \cdots \wedge d x_{n}$. Note that $f$ is a polynomial in $x$ of degree $N+1$ if $\alpha_{0} \neq 0$. In the following of this paper, we use the parameters $a=\left(a_{1}, \ldots, a_{N}\right)$ which are related to $\alpha$ by

$$
\alpha_{k}=(-1)^{k} e_{k}(a), \quad k=0,1, \ldots, N
$$

where $e_{0}(a)=1$ and $e_{k}(a)$ is the $k$-th elementary symmetric function of $a=\left(a_{1}, \ldots, a_{N}\right)$.

\section{Twisted de Rham cohomology}

Take $z \in Z_{n+1}$ and let $f$ be the polynomial of $x$ given by (2.4) and (2.5). Let $X=C^{n}$ be as in Section 2 and $\Omega^{p}(X)$ the set of $p$-forms on $X$ with polynomial coefficients. Set $\Omega^{\bullet}(X)=\bigoplus_{p} \Omega^{p}(X)$. Define the twisted exterior differentiation $d_{f}: \Omega^{\bullet}(X) \rightarrow \Omega^{\bullet}(X)$ by

$$
d_{f} \eta=d \eta+d f \wedge \eta
$$

Since $d_{f}^{2}=0$, the pair $\left(\Omega^{\bullet}(X), d_{f}\right)$ is a complex.

Definition 3.1. The cohomology group of the complex $\left(\Omega^{\bullet}(X), d_{f}\right)$ :

$$
H^{p}\left(\Omega^{\bullet}(X), d_{f}\right):=\operatorname{Ker}\left\{d_{f}: \Omega^{p}(X) \rightarrow \Omega^{p+1}(X)\right\} / d_{f} \Omega^{p-1}(X)
$$

is called the twisted de Rham cohomology group. 
Let $\mathscr{Y}(n, N-n)$ denote the set of Young diagrams $\lambda$ contained in the box of row size $n$ and column size $N-n$, namely, the diagrams whose length is less than $n$ and whose parts satisfy $\lambda_{i} \leq N-n$. Then the following result is known.

Proposition 3.2 ([11], [14]). For any $z \in Z_{n+1}$, the following holds.

(1) $H^{p}\left(\Omega^{\bullet}(X), d_{f}\right)=0$ if $p \neq 0$.

(2) $H^{n}\left(\Omega^{\bullet}(X), d_{f}\right)$ is a vector space of dimension $\left(\begin{array}{l}N \\ n\end{array}\right)$.

(3) For a Young diagram $\lambda \in \mathscr{Y}(n, N-n)$, let $s_{\lambda}(x)$ be the polynomial such that $s_{\lambda}(e(y))$ is the Schur polynomial indexed by $\lambda$, where $e(y)=\left(e_{1}(y), \ldots\right.$, $\left.e_{n}(y)\right)$ and $e_{i}(y)$ is the $i$-th elementary symmetric polynomial in $y=$ $\left(y_{1}, \ldots, y_{n}\right)$. Then the $n$-forms

$$
s_{\lambda}(x) d x_{1} \wedge \cdots \wedge d x_{n}, \quad \lambda \in \mathscr{Y}(n, N-n)
$$

give a basis of $H^{n}\left(\Omega^{\bullet}(X), d_{f}\right)$.

Remark 3.3. Proposition 3.2. says that, in the case $n=1$, we can take, as a basis of $H^{1}\left(\Omega^{\bullet}(X), d_{f}\right)$, the 1 -forms

$$
d x, x d x, \ldots, x^{N-1} d x .
$$

The basis (3.1) of $H^{n}\left(\Omega^{\bullet}(X), d_{f}\right)$ can also be obtained, for a point $z$ of the extended Veronese variety of $Z_{n+1}$, by using the exterior power structure of $H^{n}\left(\Omega^{\bullet}(X), d_{f}\right)$, see Section 4.8 .

\section{Exterior power structure of the cohomology group}

\subsection{Extended Veronese map}

It is known that the projective space $\boldsymbol{P}^{1}$ is embedded in $\boldsymbol{P}^{n}$ by the map

$$
\boldsymbol{P}^{1} \ni x_{0}: x_{1} \mapsto x_{0}^{n}: x_{0}^{n-1} x_{1}: \ldots: x_{0} x_{1}^{n-1}: x_{1}^{n} \in \boldsymbol{P}^{n}
$$

As is well known, the construction of this map can be understood from the linear algebra as follows. Let $V$ be a complex vector space of $\operatorname{dim} V=2$ and let $S^{n} V$ be the $n$-th symmetric tensor product of $V$. Then if we express the map

$$
V \rightarrow S^{n} V: v \mapsto \overbrace{v \otimes \cdots \otimes v}^{n}
$$

in terms of the basis $e_{0}, e_{1}$ of $V$ and

$$
\boldsymbol{e}_{i}=\sum_{i_{1}+\cdots+i_{n}=i} e_{i_{1}} \otimes \cdots \otimes e_{i_{n}}, \quad i=0, \ldots, n
$$

of $S^{n} V$, we have the above map (4.1) by passing to the map $\boldsymbol{P}(V) \rightarrow \boldsymbol{P}\left(S^{n} V\right)$ between the projective spaces. This map is called the Veronese map. 
Here we extend the Veronese map so that it adapts to the theory of generalized Airy function. Let $C[T]$ be the polynomial ring of one indeterminate $T$ and let $R=C[T] /\left(T^{N+2}\right)$ be the quotient ring by the ideal $\left(T^{N+2}\right)$ generated by $T^{N+2}$. Note that the group of units of $R$ is isomorphic to $H$. Instead of considering the vector space $V$, we consider the free $R$-module $\tilde{V}:=V \otimes_{C} R$, which is also a left $\mathrm{GL}(V)$-module. As a $R$-module, $\tilde{V}$ has a basis $e_{0}, e_{1}$, and as a $C$-vector space, it has a basis

$$
e_{i} \otimes T^{j}, \quad 0 \leq i \leq 1,0 \leq j \leq N+1 .
$$

Using these basis, any element $\tilde{v} \in \tilde{V}$ is expressed as

$$
\tilde{v}=\sum_{i} v_{i}(T) e_{i}=\sum_{i, j} v_{i j} e_{i} \otimes T^{j},
$$

and therefore $\tilde{V}$ can be identified with $M(2, N+2, C)$ by the correspondence

$$
\tilde{V} \ni \tilde{v} \mapsto\left(v_{i j}\right) \in M(2, N+2, C) .
$$

Now we define the map Vero: $M(2, N+2, \boldsymbol{C}) \mapsto M(n+1, N+2, \boldsymbol{C})$ as follows. Let $S^{n} \tilde{V}$ be the $n$-th symmetric tensor product as $R$-module. It is a free $R$-module with a basis $e_{i}(0 \leq i \leq n)$. Define the map Vero $: \tilde{V} \rightarrow S^{n} \tilde{V}$ by the correspondence $\tilde{v} \mapsto \tilde{v} \otimes \cdots \otimes \tilde{v}$. If $\tilde{v}=\sum_{i} v_{i}(T) e_{i}=\sum_{i j} v_{i j} e_{i} \otimes T^{j}$, then

$$
\operatorname{Vero}(\tilde{v})=\sum_{i=0}^{n} v_{0}(T)^{n-i} v_{1}(T)^{i} \boldsymbol{e}_{i}
$$

If we write

$$
v_{0}(T)^{n-i} v_{1}(T)^{i} \equiv \sum_{j=0}^{N+1} w_{i j} T^{j}, \quad\left(\bmod .\left(T^{N+2}\right)\right),
$$

then the correspondence $\left(v_{i j}\right) \mapsto\left(w_{i j}\right)$ gives the map

$$
\text { Vero : } M(2, N+2, \boldsymbol{C}) \mapsto M(n+1, N+2, \boldsymbol{C}),
$$

which we call the extended Veronese map. The image of $M(2, N+2, \boldsymbol{C})$ by Vero will be called the extended Veronese variety.

Remark 4.1. In the case $n=2, N=2$, the extended Veronese map Vero : $M(2,4, C) \mapsto M(3,4, C)$ is

$\operatorname{Vero}\left(\left(v_{i j}\right)\right)$

$$
=\left(\begin{array}{cccc}
v_{00}^{2} & 2 v_{00} v_{01} & 2 v_{00} v_{02}+v_{01}^{2} & 2 v_{00} v_{03}+2 v_{01} v_{02} \\
v_{00} v_{01} & v_{00} v_{11}+v_{01} v_{10} & v_{00} v_{12}+v_{02} v_{10}+v_{01} v_{11} & v_{00} v_{13}+v_{01} v_{12}+v_{02} v_{11}+v_{03} v_{10} \\
v_{01}^{2} & 2 v_{01} v_{11} & 2 v_{10} v_{12}+v_{11}^{2} & 2 v_{10} v_{13}+2 v_{11} v_{12}
\end{array}\right)
$$




\subsection{Exterior power structure}

Let $Y=C$ and $X$ a symmetric product of $n$ copies of $Y$, namely, $X=$ $Y^{n} / S_{n} \simeq C^{n}$, where $S_{n}$ is the symmetric group of order $n$. Let $\pi: Y^{n} \rightarrow X$ be the projection. The projection $\pi$ is realized by the map

$$
\left(y_{1}, \ldots, y_{n}\right) \mapsto\left(x_{1}, \ldots, x_{n}\right)=\left(e_{1}(y), \ldots, e_{n}(y)\right)
$$

using the elementary symmetric polynomials $e_{k}(y)$. We have $\pi^{*}: \Omega^{\bullet}(X) \rightarrow$ $\Omega^{\bullet}\left(Y^{n}\right)$. Let us define an exterior differentiation for $\Omega^{\bullet}\left(Y^{n}\right)$ so that it becomes a complex and $\pi^{*}$ becomes a chain map.

Take $z \in Z_{2}$ and let $g(y)$ be the polynomial defined by

$$
g(y)=\sum_{k=0}^{N}(-1)^{k} e_{k}(a) \theta_{N-k+1}\left(l^{-1}(\vec{y} z)\right)
$$

where $\vec{y}=(1, y)$. Let $d_{g}: \Omega^{\bullet}(Y) \rightarrow \Omega^{\bullet}(Y)$ be the exterior differetiation given by

$$
d_{g}=d+d g \wedge .
$$

By $\pi_{i}: Y^{n} \rightarrow Y$, we denote the projection to the $i$-th factor. Let $\nabla^{n} d_{g}=$ $\pi_{1}^{*} d_{g} \otimes \cdots \otimes \pi_{n}^{*} d_{g}: \Omega^{\bullet}\left(Y^{n}\right) \rightarrow \Omega^{\bullet}\left(Y^{n}\right)$ be the exterior product, namely, the exterior differentiation which satisfies, for $\varphi_{i} \in \Omega^{p_{i}}(Y)(i=1, \ldots, n)$,

$$
\left(\bigotimes^{n} d_{g}\right)\left(\pi_{1}^{*} \varphi_{1} \wedge \cdots \wedge \pi_{n}^{*} \varphi_{n}\right)=\sum_{i=1}^{n}(-1)^{p_{1}+\cdots+p_{i-1}} \pi_{1}^{*} \varphi_{1} \wedge \cdots \wedge\left(\pi_{i}^{*} d_{g} \varphi_{i}\right) \wedge \cdots \wedge \pi_{n}^{*} \varphi_{n}
$$

Lemma 4.2. For $\tilde{z}=\operatorname{Vero}(z)$, define the polynomial $f$ of $x$ by

$$
f(x)=\sum_{k=0}^{N}(-1)^{k} e_{k}(a) \theta_{N-k+1}\left(l^{-1}(\vec{x} \tilde{z})\right)
$$

Then we have $\pi^{*} f=\sum_{i=1}^{n} g\left(y_{i}\right)$.

Proof. From the definition of $\theta_{k}$, we have

$$
\theta_{k}\left(h h^{\prime}\right)=\theta_{k}(h)+\theta_{k}\left(h^{\prime}\right)
$$

for any $h, h^{\prime} \in H$. Then,

$$
\theta_{k}\left(l^{-1}\left(\vec{y}_{1} z\right)\right)+\cdots+\theta_{k}\left(l^{-1}\left(\vec{y}_{n} z\right)\right)=\theta_{k}\left(l^{-1}\left(\vec{y}_{1} z\right) \ldots l^{-1}\left(\vec{y}_{n} z\right)\right) .
$$

Moreover, from the definition of the extended Veronese map, we have

$$
l^{-1}\left(\vec{y}_{1} z\right) \ldots l^{-1}\left(\vec{y}_{n} z\right)=l^{-1}\left(\left(1, e_{1}(y), \ldots, e_{n}(y)\right) \tilde{z}\right) .
$$


Indeed, if we put

$$
l^{-1}\left(\vec{y}_{i} z\right)=\sum_{j=0}^{N+1}\left(z_{0 j}+z_{1 j} y_{i}\right) \Lambda^{j}=v_{0}(\Lambda)+v_{1}(\Lambda) y_{i}
$$

with

$$
v_{0}(\Lambda)=\sum_{j=0}^{N+1} z_{0 j} \Lambda^{j}, \quad v_{1}(\Lambda)=\sum_{j=0}^{N+1} z_{1 j} \Lambda^{j}
$$

we have

$$
\begin{aligned}
l^{-1}\left(\vec{y}_{1} z\right) \ldots l^{-1}\left(\vec{y}_{n} z\right) & =\left(v_{0}(\Lambda)+v_{1}(\Lambda) y_{1}\right) \ldots\left(v_{0}(\Lambda)+v_{1}(\Lambda) y_{n}\right) \\
& =\sum_{i=0}^{n} v_{0}(\Lambda)^{n-i} v_{1}(\Lambda)^{i} e_{i}(y) \\
& =\sum_{i=0}^{n}\left(\sum_{j=0}^{N+1} \tilde{z}_{i j} \Lambda^{j}\right) e_{i}(y) \\
& =\sum_{j=0}^{N+1}\left(\sum_{i=0}^{n} \tilde{z}_{i j} e_{i}(y)\right) \Lambda^{j} \\
& =l^{-1}\left(\left(1, e_{1}(y), \ldots, e_{n}(y)\right) \tilde{z}\right)
\end{aligned}
$$

From (4.5) and (4.6), it follows that

$$
\begin{aligned}
\pi^{*} f & =\sum_{k=0}^{N}(-1)^{k} e_{k}(a) \theta_{N-k+1}\left(l^{-1}\left(\left(1, e_{1}(y), \ldots, e_{n}(y)\right) \tilde{z}\right)\right) \\
& =\sum_{k=0}^{N}(-1)^{k} e_{k}(a) \sum_{i=1}^{n} \theta_{N-k+1}\left(l^{-1}\left(\vec{y}_{i} z\right)\right) \\
& =\sum_{i=1}^{n} g\left(y_{i}\right) .
\end{aligned}
$$

Lemma 4.3. $\pi^{*}:\left(\Omega^{\bullet}(X), d_{f}\right) \rightarrow\left(\Omega^{\bullet}\left(Y^{n}\right), \nabla^{n} d_{g}\right)$ is a chain map, and therefore it induces a homomorphism

$$
\pi^{*}: H^{\bullet}\left(\Omega^{\bullet}(X), d_{f}\right) \rightarrow H^{\bullet}\left(\Omega^{\bullet}\left(Y^{n}\right), \bigotimes^{n} d_{g}\right) .
$$

Proof. To show that $\pi^{*}$ induces a chain map, it is sufficient to show that $\pi^{*} d f=\sum_{i=1}^{n} \pi_{i}^{*} d g$. But this is shown in Lemma 4.2. 
Define an action of $S_{n}$ on $Y^{n}$ by

$$
\sigma\left(y_{1}, \ldots, y_{n}\right)=\left(y_{\sigma^{-1}(1)}, \ldots, y_{\sigma^{-1}(n)}\right), \quad \sigma \in S_{n} .
$$

It induces a chain isomorphism $\sigma^{*}:\left(\Omega^{\bullet}\left(Y^{n}\right), \nabla^{n} d_{g}\right) \rightarrow\left(\Omega^{\bullet}\left(Y^{n}\right), \bigotimes^{n} d_{g}\right)$, since $\pi^{*} d f=\sum_{i=1}^{n} \pi_{i}^{*} d g$ is invariant by $\sigma^{*}$. Thus $S_{n}$ acts on the cohomology group $H^{\bullet}\left(\Omega^{\bullet}\left(Y^{n}\right), \bigotimes^{n} d_{g}\right)$. Let $H^{\bullet}\left(\Omega^{\bullet}\left(Y^{n}\right), \bigotimes^{n} d_{g}\right)^{S_{n}}$ denote the subspace of $H^{\bullet}\left(\Omega^{\bullet}\left(Y^{n}\right), \nabla^{n} d_{g}\right)$ consisting of the invariant elements with respect to the action of $S_{n}$. It is evident that the image of the map (4.8) is contained in $H^{\bullet}\left(\Omega^{\bullet}\left(Y^{n}\right), \bigotimes^{n} d_{g}\right)^{S_{n}}$.

Proposition 4.4. The map $\pi^{*}: H^{\bullet}\left(\Omega^{\bullet}(X), d_{f}\right) \rightarrow H^{\bullet}\left(\Omega^{\bullet}\left(Y^{n}\right), \bigotimes^{n} d_{g}\right)^{S_{n}}$ is an isomorphism.

To show Proposition 4.4, we prepare a lemma. Define, for $\varphi \in \Omega^{\bullet}\left(Y^{n}\right)$,

$$
\mu(\varphi)=\sum_{\sigma \in S_{n}} \sigma^{*} \varphi
$$

Lemma 4.5. Let $\Omega^{\bullet}\left(Y^{n}\right)^{S_{n}}$ be the set of invarint elements of $\Omega^{\bullet}\left(Y^{n}\right)$ by the action of $S_{n}$. Then

(1) $\mu: \Omega^{\bullet}\left(Y^{n}\right) \rightarrow \Omega^{\bullet}\left(Y^{n}\right)$ is a multiplication by $n$ ! of the projection to $\Omega^{\bullet}\left(Y^{n}\right)^{S_{n}}$,

(2) $\mu$ defines a chain map from $\left(\Omega^{\bullet}\left(Y^{n}\right), \bigotimes^{n} d_{g}\right)$ to itself, and the image of the induced map

$$
\mu: H^{\bullet}\left(\Omega^{\bullet}\left(Y^{n}\right), \bigotimes^{n} d_{g}\right) \rightarrow H^{\bullet}\left(\Omega^{\bullet}\left(Y^{n}\right), \bigotimes^{n} d_{g}\right)
$$

is contained in $H^{\bullet}\left(\Omega^{\bullet}\left(Y^{n}\right), \bigotimes^{n} d_{g}\right)^{S_{n}}$,

(3) $\pi^{*}: \Omega^{\bullet}(X) \rightarrow \Omega^{\bullet}\left(Y^{n}\right)^{S_{n}}$ is an isomorphism.

Proof. To show the assertion (1), it is enough to show that $\mu\left(\Omega^{\bullet}\left(Y^{n}\right)\right) \subset$ $\Omega^{\bullet}\left(Y^{n}\right)^{S_{n}}$ and $\mu$ acts as a scalar multiplication by $n$ ! on an element of $\Omega^{\bullet}\left(Y^{n}\right)^{S_{n}}$. For $\varphi \in \Omega^{\bullet}\left(Y^{n}\right)$ and $\sigma \in S_{n}$, we have

$$
\sigma^{*}(\mu(\varphi))=\sigma^{*}\left(\sum_{\tau \in S_{n}} \tau^{*} \varphi\right)=\sum_{\tau \in S_{n}}(\tau \sigma)^{*} \varphi=\mu(\varphi) .
$$

This implies $\mu(\varphi) \in \Omega^{\bullet}\left(Y^{n}\right)^{S_{n}}$. For $\varphi \in \Omega^{\bullet}\left(Y^{n}\right)^{S_{n}}$, we have

$$
\mu(\varphi)=\sum_{\tau \in S_{n}} \tau^{*} \varphi=\sum_{\tau \in S_{n}} \varphi=n ! \cdot \varphi .
$$

This shows that $\mu$ is an $n$ ! times of the identity on $\Omega^{\bullet}\left(Y^{n}\right)^{S_{n}}$.

Next we show (2). For this, it is sufficient show that

$$
\mu \circ \bigotimes^{n} d_{g}=\left(\bigotimes^{n} d_{g}\right) \circ \mu .
$$


For $\varphi \in \Omega^{\bullet}\left(Y^{n}\right)$ and $\sigma \in S_{n}$, we have

$$
\begin{aligned}
\sigma^{*} \circ\left(\bigotimes^{n} d_{g}\right) \varphi & =\sigma^{*}\left(d \varphi+\left(\sum_{i=1}^{n} \pi_{i}^{*} d g\right) \wedge \varphi\right) \\
& =d\left(\sigma^{*} \varphi\right)+\left(\sum_{i=1}^{n} \pi_{i}^{*} d g\right) \wedge \sigma^{*} \varphi \\
& =\left(\bigotimes^{n} d_{g}\right) \sigma^{*} \varphi .
\end{aligned}
$$

This completes the proof of (2). The proof of the assertion (3) is easy.

Definition 4.6. The map (tf) : $\Omega^{\bullet}\left(Y^{n}\right) \rightarrow \Omega^{\bullet}(X)$ is defined by

$$
(\mathrm{tf})=\left(\pi^{*}\right)^{-1} \circ \mu \text {. }
$$

This map is well defined by virtue of Lemma 4.5 .

Since $\pi^{*}$ and $\mu$ are chain maps, (tf) is also a chain map. So it induces the map

$$
\text { (tf) }: H^{\bullet}\left(\Omega^{\bullet}\left(Y^{n}\right), \bigotimes^{n} d_{g}\right) \rightarrow H^{\bullet}\left(\Omega^{\bullet}(X), d_{f}\right) .
$$

Proof of Proposition 4.4.

The map $(1 / n !) \mu$ is an identity on $H^{\bullet}\left(\Omega^{\bullet}\left(Y^{n}\right), \bigotimes^{n} d_{g}\right)^{S_{n}}$. Indeed, let $[\varphi] \in H^{\bullet}\left(\Omega^{\bullet}\left(Y^{n}\right), \bigotimes^{n} d_{g}\right)^{S_{n}}$. Then, for any $\sigma \in S_{n}$, we have $\sigma^{*} \varphi-\varphi=\left(\bigotimes^{n} d_{g}\right) \psi_{\sigma}$ for some $\psi_{\sigma} \in \Omega^{\bullet}\left(Y^{n}\right)$. Hence

$$
\frac{1}{n !} \mu(\varphi)-\varphi=\left(\otimes^{n} d_{g}\right)\left(\frac{1}{n !} \sum_{\sigma \in S_{n}} \psi_{\sigma}\right) .
$$

Noting that $\pi^{*} \circ(\mathrm{tf})=\mu$ as a map from $\Omega^{\bullet}\left(Y^{n}\right)$ to itself and (tf) $\circ \pi^{*}=n ! \cdot$ id as a map from $\Omega^{\bullet}(X)$ to itself, the induced maps for the cohomology groups satisfy

$$
\pi^{*} \circ(\mathrm{tf})=n ! \cdot \mathrm{id}, \quad(\mathrm{tf}) \circ \pi^{*}=n ! \cdot \mathrm{id} .
$$

Thus the map $(1 / n !)(\mathrm{tf}): H^{\bullet}\left(\Omega^{\bullet}\left(Y^{n}\right), \bigotimes^{n} d_{g}\right)^{S_{n}} \rightarrow H^{\bullet}\left(\Omega^{\bullet}(X), d_{f}\right)$ gives the inverse map of $\pi^{*}: H^{\bullet}\left(\Omega^{\bullet}(X), d_{f}\right) \rightarrow H^{\bullet}\left(\Omega^{\bullet}\left(Y^{n}\right), \bigotimes^{n} d_{g}\right)^{S_{n}}$.

Define the map

$$
\kappa: \bigotimes^{n} H^{1}\left(\Omega^{\bullet}(Y), d_{g}\right) \rightarrow H^{n}\left(\Omega^{\bullet}\left(Y^{n}\right), \bigotimes^{n} d_{g}\right)
$$

by

$$
\varphi_{1} \otimes \cdots \otimes \varphi_{n} \mapsto \pi_{1}^{*} \varphi_{1} \wedge \cdots \wedge \pi_{n}^{*} \varphi_{n} .
$$

It is an isomorphism by virtue of the Künneth formula, see [9]. 
Proposition 4.7. The map (4.9) induces the isomorphism

$$
\kappa: \bigwedge^{n} H^{1}\left(\Omega^{\bullet}(Y), d_{g}\right) \rightarrow H^{n}\left(\Omega^{\bullet}\left(Y^{n}\right), \bigotimes^{n} d_{g}\right)^{S_{n}} .
$$

Proof. For $\sigma \in S_{n}$ and $\varphi_{i}=h_{i}(y) d y \in H^{1}\left(\Omega^{\bullet}(Y), d_{g}\right)(i=1, \ldots, n)$, we have

$$
\begin{aligned}
\sigma^{*} \kappa\left(\varphi_{1} \otimes \cdots \otimes \varphi_{n}\right) & =\sigma^{*}\left(\pi_{1}^{*} \varphi_{1} \wedge \cdots \wedge \pi_{n}^{*} \varphi_{n}\right) \\
& =h_{1}\left(y_{\sigma^{-1}(1)}\right) d y_{\sigma^{-1}(1)} \wedge \cdots \wedge h_{n}\left(y_{\sigma^{-1}(n)}\right) d y_{\sigma^{-1}(n)} \\
& =(\operatorname{sgn} \sigma) h_{\sigma(1)}\left(y_{1}\right) d y_{1} \wedge \cdots \wedge h_{\sigma(n)}\left(y_{n}\right) d y_{n} \\
& =(\operatorname{sgn} \sigma) \pi_{1}^{*} \varphi_{\sigma(1)} \wedge \cdots \wedge \pi_{n}^{*} \varphi_{\sigma(n)} .
\end{aligned}
$$

Define the automorphism $\sigma^{\#}: \bigotimes^{n} H^{1}\left(\Omega^{\bullet}(Y), d_{g}\right) \rightarrow \bigotimes^{n} H^{1}\left(\Omega^{\bullet}(Y), d_{g}\right)$ by

$$
\sigma^{\#}\left(\varphi_{1} \otimes \cdots \otimes \varphi_{n}\right)=(\operatorname{sgn} \sigma) \varphi_{\sigma(1)} \otimes \cdots \otimes \varphi_{\sigma(n)} .
$$

Then (4.10) implies that the following diagram is commutative:

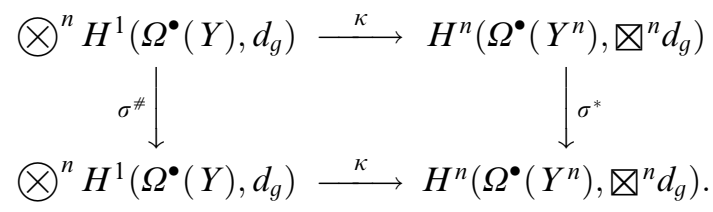

Thus, if we put $\mu^{\#}=\sum_{\sigma \in S_{n}} \sigma^{\#}$, then we have

$$
\begin{aligned}
\kappa\left(\bigwedge^{n} H^{1}\left(\Omega^{\bullet}(Y), d_{g}\right)\right) & =\kappa \mu^{\#}\left(\bigotimes^{n} H^{1}\left(\Omega^{\bullet}(Y), d_{g}\right)\right) \\
& =\mu^{*} \kappa\left(\bigotimes^{n} H^{1}\left(\Omega^{\bullet}(Y), d_{g}\right)\right) \\
& =\mu^{*}\left(H^{n}\left(\Omega^{\bullet}\left(Y^{n}\right), \bigotimes^{n} d_{g}\right)\right) \\
& =H^{n}\left(\Omega^{\bullet}\left(Y^{n}\right), \bigotimes^{n} d_{g}\right)^{S_{n}} .
\end{aligned}
$$

Thus the proof of the proposition is completed.

Combining Propositions 4.4 and 4.7, we have the isomorphisms

$$
H^{n}\left(\Omega^{\bullet}(X), d_{f}\right) \stackrel{\pi^{*}}{\rightarrow} H^{n}\left(\Omega^{\bullet}\left(Y^{n}\right), \bigotimes^{n} d_{g}\right) \stackrel{S_{n}}{\stackrel{\kappa}{\leftarrow}} \bigwedge^{n} H^{1}\left(\Omega^{\bullet}(Y), d_{g}\right) .
$$

Thus we have the following theorem. 
Theorem 4.8. Let $z \in Z_{2}$ and $\tilde{z} \in Z_{n+1}$ be such that $\tilde{z}=\operatorname{Vero}(z)$. And let $f(x)$ and $g(y)$ be the polynomials of $x=\left(x_{1}, \ldots, x_{n}\right)$ and $y$ defined by (4.3) and (4.2), respectively. Then we have the isomorphism

$$
\left(\pi^{*}\right)^{-1} \circ \kappa: \bigwedge^{n} H^{1}\left(\Omega^{\bullet}(Y), d_{g}\right) \rightarrow H^{n}\left(\Omega^{\bullet}(X), d_{f}\right) .
$$

\subsection{Explicit basis of the cohomology group}

Let $z \in Z_{2}$ and $g(y)$ be the polynomial as in (4.2). Then we have the following.

Proposition 4.9 ([13]). The cohomology group $H^{1}\left(\Omega^{\bullet}(Y), d_{g}\right)$ has a basis as C-vector space

$$
\phi_{i}=d \theta_{i}\left(l^{-1}(\vec{y} z)\right), \quad 1 \leq i \leq N .
$$

In the following, we omit $l^{-1}$ in the expression $d \theta_{i}\left(l^{-1}(\vec{y} z)\right)$ and denote it simply as $d \theta_{i}(\vec{y} z)$ when there is no fear of confusion.

Next we give a basis of the cohomology group $H^{n}\left(\Omega^{\bullet}(X), d_{f}\right)$, when $f$ is defined by (4.3) for $\tilde{z} \in Z_{n+1}$ belonging to the extended Veronese variety.

Proposition 4.10. Let $\tilde{z} \in Z_{n+1}$ be in the image of Vero. For any $\lambda=$ $\left(\lambda_{1}, \ldots, \lambda_{n}\right) \in \mathscr{Y}(n, N-n)$, put

$$
\phi_{\lambda}=d \theta_{\lambda_{1}+n}(\vec{x} \tilde{z}) \wedge d \theta_{\lambda_{2}+n-1}(\vec{x} \tilde{z}) \wedge \cdots \wedge d \theta_{\lambda_{n}+1}(\vec{x} \tilde{z}) .
$$

Then $\left\{\phi_{\lambda} \mid \lambda \in \mathscr{Y}(n, N-n)\right\}$ gives a basis for both $H^{n}\left(\Omega^{\bullet}(X), d_{f}\right)$ and $H^{n}\left(\Omega^{\bullet}(X), d_{-f}\right)$.

Proof. Let $\square$ denotes the exterior product in $\bigwedge^{n} H^{1}\left(\Omega^{\bullet}(Y), d_{g}\right)$. Then the map $\left(\pi^{*}\right)^{-1} \circ \kappa$ in Theorem 4.8 is the correspondence

$$
\varphi_{1} \square \cdots \square \varphi_{n} \mapsto(\mathrm{tf})\left(\pi_{1}^{*} \varphi_{1} \wedge \cdots \wedge \pi_{n}^{*} \varphi_{n}\right) .
$$

Noting that $(\mathrm{tf})=\left(\pi^{*}\right)^{-1} \circ \mu$, we compute

$$
\begin{aligned}
\mu\left(\pi_{1}^{*} \varphi_{1} \wedge \cdots \wedge \pi_{n}^{*} \varphi_{n}\right) & =\mu\left(\varphi_{1}\left(y_{1}\right) \wedge \cdots \wedge \varphi_{n}\left(y_{n}\right)\right) \\
& =\sum_{\sigma \in S_{n}} \varphi_{1}\left(y_{\sigma^{-1}(1)}\right) \wedge \cdots \wedge \varphi_{n}\left(y_{\sigma^{-1}(n)}\right) \\
& =\sum_{\sigma \in S_{n}}(\operatorname{sgn} \sigma) \varphi_{\sigma(1)}\left(y_{1}\right) \wedge \cdots \wedge \varphi_{\sigma(n)}\left(y_{n}\right) .
\end{aligned}
$$

We apply this to the forms $\left(\varphi_{1}, \ldots, \varphi_{n}\right)=\left(d \theta_{j_{1}}(\vec{y} z), \ldots, d \theta_{j_{n}}(\vec{y} z)\right)$ with $N \geq$ $j_{1}>\cdots>j_{n} \geq 1$ to get

$$
\mu\left(d \theta_{j_{1}}\left(\vec{y}_{1} z\right) \wedge \cdots \wedge d \theta_{j_{n}}\left(\vec{y}_{n} z\right)\right)=\sum_{\sigma \in S_{n}}(\operatorname{sgn} \sigma) d \theta_{j_{\sigma(1)}}\left(\vec{y}_{1} z\right) \wedge \cdots \wedge d \theta_{j_{\sigma(n)}}\left(\vec{y}_{n} z\right)
$$


Next we compute $\pi^{*}\left(d \theta_{j_{1}}(\vec{x} \tilde{z}) \wedge \cdots \wedge d \theta_{j_{n}}(\vec{x} \tilde{z})\right)$. Noting that the identity

$$
\pi^{*}\left(\theta_{j_{k}}(\vec{x} \tilde{z})\right)=\theta_{j_{k}}\left(\vec{y}_{1} z\right)+\cdots+\theta_{j_{k}}\left(\vec{y}_{n} z\right)
$$

holds, which is given in the proof of Lemma 4.2, we have

$$
\pi^{*}\left(d \theta_{j_{k}}(\vec{x} \tilde{z})\right)=\sum_{i=1}^{n} d \theta_{j_{k}}\left(\vec{y}_{i} z\right) .
$$

Then

$$
\begin{aligned}
\pi^{*}\left(d \theta_{j_{1}}(\vec{x} \tilde{z}) \wedge \cdots \wedge d \theta_{j_{N}}(\vec{x} \tilde{z})\right) & =\left(\sum_{i_{1}=1}^{n} d \theta_{j_{1}}\left(\vec{y}_{i_{1}} z\right)\right) \wedge \cdots \wedge\left(\sum_{i_{n}=1}^{n} d \theta_{j_{n}}\left(\vec{y}_{i_{n}} z\right)\right) \\
& =\sum_{\sigma \in S_{n}} d \theta_{j_{1}}\left(\vec{y}_{\sigma^{-1}(1)} z\right) \wedge \cdots \wedge d \theta_{j_{n}}\left(\vec{y}_{\sigma^{-1}(n)} z\right) \\
& =\sum_{\sigma \in S_{n}}(\operatorname{sgn} \sigma) d \theta_{j_{\sigma(1)}}\left(\vec{y}_{1} z\right) \wedge \cdots \wedge d \theta_{j_{\sigma(n)}}\left(\vec{y}_{n} z\right) .
\end{aligned}
$$

Thus, from (4.12), (4.13) and (4.14), we get

$$
d \theta_{j_{1}}(\vec{x} \tilde{z}) \wedge \cdots \wedge d \theta_{j_{n}}(\vec{x} \tilde{z})=\left(\pi^{*}\right)^{-1} \circ \kappa\left(d \theta_{j_{1}}(\vec{y} z) \square \cdots \square d \theta_{j_{n}}(\vec{y} z)\right) .
$$

Defining the Young diagram $\lambda=\left(\lambda_{1}, \ldots, \lambda_{n}\right)=\left(j_{1}-n, j_{2}-(n-1), \ldots\right.$, $\left.j_{n-1}-2, j_{n}-1\right) \in \mathscr{Y}(n, N-n)$, we see that

$$
\phi_{\lambda}=\left(\pi^{*}\right)^{-1} \circ \kappa\left(d \theta_{\lambda_{1}+n}(\vec{y} z) \square \cdots \square d \theta_{\lambda_{n}+1}(\vec{y} z)\right) .
$$

\section{Cohomological intersection number}

\subsection{Definition of intersection number}

First we briefly recall the definition of the intersection number for the twisted de Rham cohomologies due to Iwasaki [9], [11]. This comes from the duality between $H^{n}\left(\Omega^{\bullet}(X), d_{f}\right)$ and $H^{n}\left(\Omega^{\bullet}(X), d_{-f}\right)$. We have an isomorphism

$$
H^{n}\left(\Omega^{\bullet}(X), d_{ \pm f}\right) \stackrel{\sim}{\rightarrow} H^{n}\left(\Omega^{\bullet}\left(Y^{n}\right), d_{ \pm f}\right)^{S_{n}} .
$$

Let $\mathscr{S}^{\bullet}\left(Y^{n}\right)$ and $\mathscr{T}^{\bullet}\left(Y^{n}\right)$ be the set of differential forms of Schwartz class on $Y^{n}$ and the set of tempered currents on $Y^{n}$, respectively. Then the natural $S_{n}$-equivariant chain map

$$
\left(\Omega^{\bullet}\left(Y^{n}\right), d_{ \pm f}\right) \hookrightarrow\left(\mathscr{T}^{\bullet}\left(Y^{n}\right), d_{ \pm f}\right) \hookleftarrow\left(\mathscr{S}^{\bullet}\left(Y^{n}\right), d_{ \pm f}\right)
$$

induces the $S_{n}$-equivariant isomorphims

$$
H^{n}\left(\Omega^{\bullet}\left(Y^{n}\right), d_{ \pm f}\right) \stackrel{\sim}{\rightarrow} H^{n}\left(\mathscr{T}^{\bullet}\left(Y^{n}\right), d_{ \pm f}\right) \stackrel{\sim}{\leftarrow} H^{n}\left(\mathscr{S} \bullet\left(Y^{n}\right), d_{ \pm f}\right) .
$$


On the other hand, from the duality between $\mathscr{S}^{\bullet}\left(Y^{n}\right)$ and $\mathscr{T}^{\bullet}\left(Y^{n}\right)$, we have the $S_{n}$-equivariant duality

$$
H^{n}\left(\mathscr{S}^{\bullet}\left(Y^{n}\right), d_{f}\right) \times H^{n}\left(\mathscr{T}^{\bullet}\left(Y^{n}\right), d_{-f}\right) \rightarrow \boldsymbol{C} .
$$

Combining this duality and the isomorphism (5.1), we have the $S_{n}$-equivalent duality

$$
H^{n}\left(\Omega^{\bullet}\left(Y^{n}\right), d_{f}\right) \times H^{n}\left(\Omega^{\bullet}\left(Y^{n}\right), d_{-f}\right) \rightarrow C,
$$

which yields the duality

$$
H^{n}\left(\Omega^{\bullet}(X), d_{f}\right) \times H^{n}\left(\Omega^{\bullet}(X), d_{-f}\right) \rightarrow \boldsymbol{C} .
$$

Theorem $5.1([9])$. For $\phi^{ \pm} \in H^{n}\left(\Omega^{\bullet}\left(Y^{n}\right), d_{ \pm f}\right)$, the intersection number $\left\langle\phi^{+}, \phi^{-}\right\rangle$is given by

$$
\left\langle\phi^{+}, \phi^{-}\right\rangle=\frac{1}{(2 \pi)^{n}} \int_{Y^{n}} \psi^{+} \wedge \phi^{-}
$$

where $\psi^{+} \in H^{n}\left(\mathscr{S}^{\bullet}\left(Y^{n}\right), d_{f}\right)$ is the class corresponding to $\phi^{+}$by the isomorphims (5.1).

\subsection{Main theorem}

Theorem 5.2. Let $z \in Z_{n+1}$ belong to the extended Veronese variety and let $\left\{\phi_{\lambda}^{ \pm} \mid \lambda \in \mathscr{Y}(n, N-n)\right\}$ be the basis of $H^{n}\left(\Omega^{\bullet}(X), d_{ \pm f}\right)$ given in Proposition 4.10. Then the cohomological intersection numbers are given by

$$
\left\langle\phi_{\lambda}^{+}, \phi_{\mu}^{-}\right\rangle=n !(-1)^{n(n-1) / 2} s_{\lambda / \check{\mu}}(a) .
$$

Here $\breve{\mu}=\left(N-n-\mu_{n}, N-n-\mu_{n-1}, \ldots, N-n-\mu_{1}\right)$ denotes the complementary Young diagram of $\mu$ in $\mathscr{Y}(n, N-n)$, and $s_{\lambda / \tilde{\mu}}(a)$ denotes the skew-Schur polynomial of a for the diagrams $\lambda$ and $\check{\mu}$.

\section{Proof of the main theorem}

\subsection{One dimensional particular case}

First we consider the case where $z \in Z_{2}$ has the form

$$
\left(\begin{array}{ccccc}
1 & 0 & 0 & \cdots & 0 \\
0 & 1 & \zeta_{2} & \cdots & \zeta_{N+1}
\end{array}\right)
$$

Let $f$ be the polynomial

$$
f=\sum_{k=0}^{N}(-1)^{k} e_{k}(a) \theta_{N-k+1}(\vec{x} z), \quad \vec{x}=(1, x),
$$


for $z \in Z_{2}$ given by (6.1). Since $z$ has the form (6.1), by (2.2), we see that $\theta_{m}(\vec{x} z)$ is a polynomial of $x$ of degree $m$ and the coefficient of $x^{m}$ is $(-1)^{m} / m$.

Lemma 6.1 ([17]). Let $z \in Z_{2}$ be given by (6.1). We can choose the coefficients $w_{1}, w_{2}, \ldots, w_{N}$ so that the Laurent polynomial

$$
w=-x\left(1+w_{1} x^{-1}+w_{2} x^{-2}+\cdots+w_{N} x^{-N}\right)
$$

has the property

$$
\theta_{m}(\vec{x} z)=-\frac{1}{m}\left(w^{m}\right)_{+}, \quad 1 \leq m \leq N+1,
$$

where $\left(w^{m}\right)_{+}$is the polynomial part of terms of degree greater than 1 of the Laurent polynomial $w^{m}$.

Note that we can use $w$ as the coordinates on $X$ near $x=\infty$ instead of $x$. Following the idea of [11], we compute the intersection number for $\phi^{+} \in$ $H^{1}\left(\Omega^{\bullet}(X), d_{f}\right)$ and $\phi^{-} \in H^{1}\left(\Omega^{\bullet}(X), d_{-f}\right)$ in the following steps.

- Solve the equation

$$
d_{f} \psi^{+}=\phi^{+}
$$

in the form of formal power series in $w: \psi^{+}=\sum_{k=1}^{\infty} v_{k}^{+} w^{-k}$.

- Let $\xi^{+}$be a smooth function on $X$ such that its Taylor expansion at $w=\infty$ coincides with $\psi^{+}$. Put $\varphi^{+}=\phi^{+}-d_{f} \xi^{+}$. Then $\varphi^{+}$defines the class in $H^{1}\left(\mathscr{S}^{\bullet}(X), d_{f}\right)$ which corresponds to $\phi^{+}$via the isomorphism (5.1).

- Compute the intersection number $\left\langle\phi^{+}, \phi^{-}\right\rangle=\left\langle\varphi^{+}, \phi^{-}\right\rangle$using the residue calculus.

First we solve the differential equation in a formal power series. Put

$$
\phi^{ \pm}=\sum_{k=1}^{N} u_{k}^{ \pm} d \theta_{N-k+1}(\vec{x} z) .
$$

Then from (6.2), we have

$$
\begin{aligned}
\phi^{ \pm} & =-\sum_{k=1}^{N} \frac{u_{k}^{ \pm}}{N-k+1} d\left(w^{N-k+1}\right)_{+} \\
& =-\sum_{k=1}^{N} u_{k}^{ \pm} w^{N-k} d w+(\text { terms of negative powers in } w) d w
\end{aligned}
$$

If the formal power series $\psi^{+}=\sum_{k=1}^{\infty} v_{k}^{+} w^{-k}$ satisfies the equation (6.3), we have 


$$
\begin{gathered}
\sum_{j=1}^{\infty}(-j) v_{j}^{+} w^{-j-1} d w+\left(\sum_{k=0}^{N}(-1)^{k} e_{k}(a) \theta_{N-k+1}(\vec{x} z)\right) \wedge\left(\sum_{j=1}^{\infty} v_{j}^{+} w^{-j}\right) \\
=-\sum_{k=1}^{N} u_{k}^{ \pm} w^{N-k} d w+(\text { terms of negative powers in } w) d w
\end{gathered}
$$

Equating the coefficients of nonnegative powers of both sides, we have

$$
\sum_{j=0}^{k-1} e_{j}(a) v_{k-j}^{+}=u_{k}^{+}, \quad k=1, \ldots, N .
$$

Solving this equation with respect to $v_{k}^{+}$,

$$
v_{k}^{+}=\sum_{j=0}^{k-1} h_{j}(a) u_{k-j}^{+}, \quad k=1, \ldots, N,
$$

where $h_{j}(a)$ is the complete symmetric function of $a$ of degree $j$.

Lemma 6.2. Let $\phi^{ \pm} \in H^{1}\left(\Omega^{\bullet}(X), d_{ \pm f}\right)$ be as in (6.4). Then

$$
\left\langle\phi^{+}, \phi^{-}\right\rangle=\operatorname{Res}_{w=\infty}\left(\psi^{+} \phi^{-}\right)=\sum_{j=1}^{N} \sum_{k=1}^{N} h_{N+1-j-k}(a) u_{j}^{+} u_{k}^{-} .
$$

Proof. If we use the coordinate $w$ of $X$ at $x=\infty$ the proof proceeds in the same way as that of [11].

Proof of Theorem 5.2 in one dimensional particular case. Let $\lambda=(p), \mu=$ $(q) \in \mathscr{Y}(1, N-1)$. Apply Lemma 6.2 to $\phi^{+}=\phi_{\lambda}^{+}=d \theta_{p+1}(\vec{x} z)$ and $\phi^{-}=\phi_{\mu}^{-}=$ $d \theta_{q+1}(\vec{x} z)$. Then

in (6.4) and

$$
u_{k}^{+}=\delta_{k, N-p}, \quad u_{k}^{-}=\delta_{k, N-q}
$$

$$
\left\langle\phi_{\lambda}^{+}, \phi_{\mu}^{-}\right\rangle=h_{p+q+1-N}(a)=s_{\lambda / \check{\mu}}(a) .
$$

Here we used the relation between the complete symmetric polynomials and the skew Schur functions, see [20, p. 70]. This complete the proof of Main theorem in the case where $n=1$ and $z$ has the form (6.1).

\subsection{Reduction to the particular case}

In the above subsection, we proved the Main theorem in the case $n=1$ for $z$ having the form (6.1). In this subsection we complete the proof for general $z \in Z_{2}$. The following lemma is easily checked by computation, and hence the proof is omitted. 
Lemma 6.3. For $z \in Z_{2}$, there exist $g \in \mathrm{GL}(2, C)$ and $h \in H$ such that

$$
g z h=\left(\begin{array}{ccccc}
1 & 0 & 0 & \cdots & 0 \\
0 & 1 & \zeta_{2} & \cdots & \zeta_{N+1}
\end{array}\right) .
$$

Thus it is sufficient to show that the intersection numbers are invariant by the action of an element of $\operatorname{GL}(2, \boldsymbol{C}) \times H$ which maps $Z_{2}$ to $Z_{2}$. The following Lemma is the consequence of the identity (4.4). It assures that the intersection number $\left\langle\phi^{+}, \phi^{-}\right\rangle$is invariant by the action of $H$.

Lemma 6.4. For any $h \in H$, we have

$$
\begin{aligned}
d \theta_{i}\left(l^{-1}(\vec{x} z h)\right) & =d \theta_{i}\left(l^{-1}(\vec{x} z)\right), \quad i=1, \ldots, N, \\
d f(x, z h, \alpha) & =d f(x, z, \alpha) .
\end{aligned}
$$

It is easily seen that $g \in \operatorname{GL}(2, \boldsymbol{C})$, which induces the automorphism $z \mapsto g z$ of $Z_{2}$, has the form

$$
g=\left(\begin{array}{ll}
a & b \\
0 & c
\end{array}\right) .
$$

The action of $g$ on $Z_{2}$ is realized by the change of coodinates of $X$

$$
L_{g}: x \mapsto b / a+(c / a) x .
$$

Since the definition of the intersection number is independent of the choice of coodinates of $X$, we have

Lemma 6.5. Let $g \in \mathrm{GL}(2, \boldsymbol{C})$ be as in (6.8). Then

$$
\left\langle L_{g}^{*} \phi^{+}, L_{g}^{*} \phi^{-}\right\rangle=\left\langle\phi^{+}, \phi^{-}\right\rangle
$$

Thus the proof of Theorem 5.2 for general $z \in Z_{2}$ is reduced to the case given in Section 6.1, and hence the proof of Theorem 5.2 for $n=1$ is completed.

\subsection{Multidimensional case}

Combining the argument of Lemma 4 of [11] and that in Section 4.2, we have the following lemma, whose proof we omit.

Lemma 6.6. Let $\varphi_{1}^{ \pm} \square \cdots \square \varphi_{n}^{ \pm}$correspond to $\phi^{ \pm}$via the isomorphism (4.11) in Theorem 4.8. Then we have

$$
\left\langle\phi^{+}, \phi^{-}\right\rangle=n ! \operatorname{det}\left(\left\langle\varphi_{j}^{+}, \varphi_{k}^{-}\right\rangle\right)
$$

Proof of Theorem 5.2 for $n$ general.

The argument is the same as in [11]. For $\lambda=\left(\lambda_{1}, \ldots, \lambda_{n}\right), \quad \mu=$ $\left(\mu_{1}, \ldots, \mu_{n}\right) \in \mathscr{Y}(n, N-n)$, we take $\phi_{\lambda}^{+} \in H^{n}\left(\Omega^{\bullet}(X), d_{f}\right)$ and $\phi_{\mu}^{-} \in H^{n}\left(\Omega^{\bullet}(X)\right.$, 
$\left.d_{-f}\right)$. Then, as we saw in (4.16) in the proof of Proposition 4.10, $\phi_{\lambda}^{+}$and $\phi_{\mu}^{-}$ correspond, respectively, to $\varphi_{1}^{+} \square \cdots \square \varphi_{n}^{+}$and $\varphi_{1}^{-} \square \cdots \square \varphi_{n}^{-}$with

$$
\varphi_{j}^{+}=d \theta_{\lambda_{j}+n-j+1}(\vec{y} z), \quad \varphi_{k}^{-}=d \theta_{\mu_{k}+n-k+1}(\vec{y} z) .
$$

Then, from Theorem 5.2 in the case of $n=1$, we have

$$
\begin{aligned}
\left\langle\varphi_{j}^{+}, \varphi_{k}^{-}\right\rangle & =h_{\left(\lambda_{j}+n-j\right)+\left(\mu_{k}+n-k\right)+1-N}(a) \\
& =h_{\lambda_{j}-\check{\mu}_{n+1-k}-j+(n+1-k)}(a),
\end{aligned}
$$

see also (6.7). Here we used $\mu_{k}=N-n-\check{\mu}_{n+1-k}$. Then

$$
\begin{aligned}
\left\langle\phi_{\lambda}^{+}, \phi_{\mu}^{-}\right\rangle & =n ! \operatorname{det}\left(h_{\lambda_{j}-\check{\mu}_{n+1-k}-j+(n+1-k)}(a)\right) \\
& =n !(-1)^{n(n-1) / 2} \operatorname{det}\left(h_{\lambda_{j}-\check{\mu}_{k}-j+k}(a)\right) \\
& =n !(-1)^{n(n-1) / 2} s_{\lambda / \check{\mu}}(a)
\end{aligned}
$$

by virtue of the Jacobi-Trudi identity for the skew-Schur polynomials ([20, pp. 70]). This complete the proof of Theorem 5.2 for $n$-dimensional case.

\section{References}

[1] Aomoto, K., Les équation aux différences linéaires et les intégrales des fonctions multiformes, J. Fac. Sci. Univ. Tokyo, Sect. IA math., 22 (1975), 271-297.

[2] Aomoto, K. and Kita, M., Hypergeometric functions (in Japanese), Springer-Verlag, Tokyo, 1994.

[ 3 ] Arnold, V., Varchenko, A. and Goussein-Zadé, S., Singularités des applications différentiables, Vol. 1, 2, Mir Moscou, 1986.

[4] Erdélyi et al., Higher transcendental functions, Vol. 1, MacGraw-Hill Book Company, Inc., New York-Tronto-London, 1953.

[ 5 ] Gelfand, I. M., General theory of hypergeometric functions, Dokl. Akad. Nauk. SSSR, 288 (1986), 14-18; English transl.: Soviet Math. Dokl., 33 (1986), 9-13.

[6] Gelfand, I. M., Retahk, V. S. and Serganova, V. V., Generalized Airy funcitons, Schubert cells, and Jordan groups, Dokl. Akad. Nauk. SSSR, 298 (1988), 17-21; English transl.: Soviet Math. Dokl., 37 (1988), 8-12.

[ 7 ] Ishiura, S. and Noumi, M., Calculus of the Gauss-Manin system of type $A_{l}$, I, Proc. Japan Acad., 58 (1982), 13-16.

[ 8 ] Ishiura, S. and Noumi, M., Calculus of the Gauss-Manin system of type $A_{l}$, II, Proc. Japan Acad., 58 (1982), 62-65.

[9] Iwasaki, K., Isolated singularity, Witten's Laplacian and duality for twisted de Rham cohomology, Comm. Partial Differential Equations, 28 (2003), 61-82.

[10] Iwasaki, K., Kimura, H., Shimomura, S. and Yoshida, M., From Gauss to Painlevé, Aspects Math., E16, Friedr, Vieweg \& Sohn, Braunschweig, 1991.

[11] Iwasaki, K. and Matsumoto, K., Intersection matrix of a generalized Airy function in terms of skew-Schur polynomials, Proc. Japn Acad., 76 (2000), 135-140.

[12] Iwasaki, K. and Kita, M., Exterior power structure on the twisted de Rham cohomology of the complements of real Veronese arrangements, J. Math. Pures Appl., 75 (1995), 69-84. 
[13] Kimura, H., On rational de Rham cohomology associated with the generalized confluent hypergeometric functions I, $\boldsymbol{P}^{1}$ case, Royal Soc. Edinburgh, 127A (1997), 145-155.

[14] Kimura, H., On rational de Rham cohomology associated with the generalized Airy functions, Ann. Scuola Norm. Sup. Pisa, 24 (1997), 351-366.

[15] Kimura, H., On the homology group associated with the general Airy integral, Kumamoto J. Math., 10 (1997), 11-29.

[16] Kimura, H. and Koitabashi, T., Normalizer of maximal abelian subgroups of $G L(n)$ and the general hypergeometric functions, Kumamoto J. Math., 9 (1996), 13-43.

[17] Kimura, H. and Taneda, M., Analogue of flat basis and cohomological intersection numbers for general hypergeometric functions, J. Math. Sci. Univ. Tokyo, 6 (1999), 415-436.

[18] Kita, M. and Yoshida, M., Intersection theory for twisted cycles I, II, Math. Nachr., 166 (1994), 287-304, 168 (1994), 171-190.

[19] Majima, H., Matsumoto, K. and Takayama, N., Quadratic relations for confluent hypergeometric functions, Tohoku Math. J., 52 (2000), 489-513.

[20] Macdonald, I. G., Symmetric Functions and Hall Polynomials, 2nd Edition, Oxford Science Publications, Clarendon Press, 1995.

[21] Matsumoto, K., Quadratic identities for hypergeometric series of type $(k, l)$, Kyushu J. of Math., 2 (1994), 335-345.

[22] Pham, F., Vanishing homologies and the $n$-variable saddle point method, Proceeding of the Symposia in Pure and Applied Math., Am. Math. Soc., 40 (1983), 319-334.

[23] Pham, F., La déscente des cols par les onglets de Lefschetz, avec vues sur Gauss-Manin, in Systèmes différentiels et singularités Astérisque, 130 (1985), 11-47.

[24] Saito, K., Yano, T., and Sekiguchi, J., On a certain generator system of the ring of invariants of a finite reflection group, Comm. in Algebra, 8 (1980), 373-408.

\author{
nuna adreso: \\ Irina Basalaeva \\ E-mail: kakatusina@hotmail.com \\ Hironobu Kimura \\ Department of Mathematics \\ Faculty of Sciences \\ Kumamoto University \\ Kumamoto 860-8555 \\ Japan \\ E-mail: hiro@sci.kumamoto-u.ac.jp \\ Takashi Nakazuka \\ Department of Mathematics \\ Faculty of Sciences \\ Kumamoto University \\ Kumamoto 860-8555 \\ Japan \\ E-mail: nakaduka@sci.kumamoto-u.ac.jp
}

(Ricevita la 31-an de oktobro, 2003)

(Reviziita la 18-an de marto, 2005) 